Internist 2018 $59: 151-158$

https://doi.org/10.1007/s00108-017-0373-2

Online publiziert: 11. Januar 2018

(c) Der/die Autor(en) 2018. Dieser Artikel ist eine Open-Access-Publikation.

\section{Redaktion}

W. Hiddemann, München

M. Reincke, München

CrossMark

D. De Simoni $\cdot$ R. Höftberger

Klinisches Institut für Neurologie, Medizinische Universität Wien, Wien, Österreich

\title{
Paraneoplastische neurologische Syndrome
}

\section{Eine aktuelle Zusammenfassung}

eine neuronale Dysfunktion auslösen. Oberflächenrezeptorantikörper können in Patienten mit wie auch ohne Tumorerkrankung auftreten und sprechen in der Regel gut auf eine Immuntherapie und falls erforderlich Tumortherapie an [2].

\section{Diagnose}

Die Diagnose von PNNS beruht auf der klinischen Präsentation, dem Nachweis eines Tumors und der Antikörpertestung, bei gleichzeitigem Ausschluss etwaiger anderer tumor- oder therapiebedingter Ursachen. Eine Auflistung der Hauptmerkmale sowie assoziierter Tumoren der häufigsten PNNS-Erkrankungen findet sich in - Tab. 2. Hilfreiche paraklinische Tests sind

- die Bildgebung (Magnetresonanztomographie [MRT]; „fluid-attenuated inversion recovery" [FLAIR] und T2gewichtete Sequenzen),

- Liquoruntersuchungen (erhöhte Zellzahl, erhöhtes Eiweiß, intrathekale Immunglobulinsynthese) und - die Elektroenzephalographie (EEG).

Empfehlungen zum Tumorscreening wurden von der EFNS Task Force zusammengefasst [7]. Um ein neurologisches Syndrom bei fehlendem Nachweis von onkoneuronalen Antikörpern als wahrscheinliches oder definitives PNNS bezeichnen $\mathrm{zu}$ können, dürfen definitionsgemäß zwischen Auftreten der neurologischen Symptomatik und Detektion eines Tumors maximal 5 Jahre liegen [3].

Da die Labor-Bestimmung spezifischer Antikörper mitunter länger dauern kann, muss die Therapieentscheidung oft noch vor Bestätigung des spezifischen PNNS getroffen werden. Aus diesem Grund wurden klinische Kriterien definiert, welche die Diagnose einer wahrscheinlichen oder definitiven Autoimmunenzephalitis noch vor Eintreffen der Antikörpertestergebnisse ermöglichen [4].

\section{Krankheitsbilder des zentralen Nervensystems}

\section{Paraneoplastische Enzephalo- myelitis}

Die paraneoplastische Enzephalomyelitis (PEM) ist klinisch durch eine akut bis subakut auftretende neurologische Dysfunktion gekennzeichnet, die unterschiedliche Areale des zentralen Nervensystems, die Spinalganglien und das autonome Nervensystem erfassen kann. Am häufigsten sind

- der Hippocampus,

- Hirnstamm,

- Basalganglien,

- Kleinhirn,

- Rückenmark,

- Spinalganglien (sensorische Neuronopathie) und

- das autonome Nervensystem (z. B. orthostatische Hypotension, gastrointestinale Pseudoobstruktion, Herzrhythmusstörungen)

betroffen. Die Diagnose einer PEM sollte dann erwogen werden, wenn die Hauptsymptomatik aus der Beteiligung von $\geq 2$ der zuvor aufgelisteten Areale resultiert [6]. 
Tab. 1 Klassische und nichtklassische PNNS

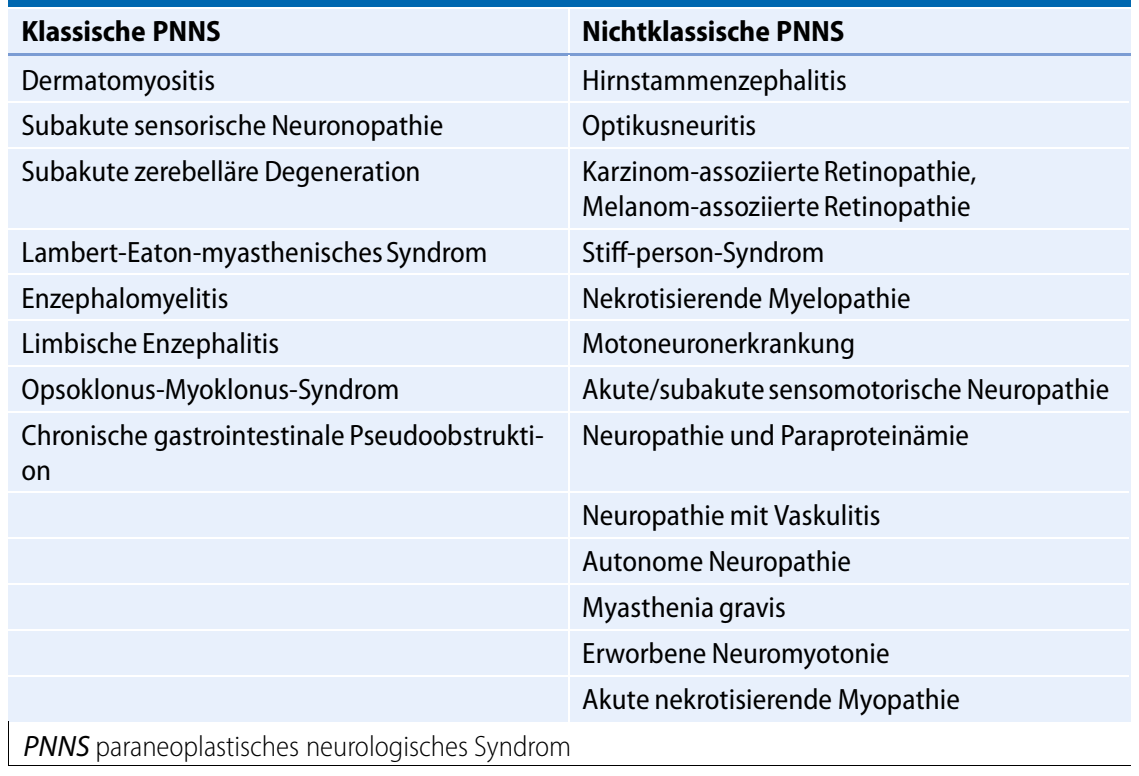

Die PEM kann mit klassischen onkoneuronalen Antikörpern gegen $\mathrm{Hu}, \mathrm{Ri}$, Ma1/2, CV2/CRMP5 und Amphiphysin assoziiert sein, wobei die klinische Präsentation abhängig von der zugrunde liegenden Autoimmunität variieren kann. Zum Beispiel findet sich bei der PEM assoziiert mit Anti-Hu-Antikörpern oftmals eine Beteiligung des unteren Hirnstamms (Pons und Medulla oblongata), weiterhin kann das Spinalganglion oder der Plexus myentericus mitbetroffen sein und eine sensorische Neuronopathie bzw. intestinale Pseudoobstruktion auftreten. Patienten mit Anti-CV2/CRMP5-Antikörpern entwickeln mitunter eine Chorea, Uveitis oder Optikusneuropathie, in manchen Fällen kann auch das periphere Nervensystem beteiligt sein, meist in Form einer sensomotorischen Neuropathie [6].

\section{》) Die PEM assoziiert mit onkoneuronalen Antikörpern hat eine ungünstige Prognose}

Die PEM assoziiert mit onkoneuronalen Antikörpern ist mit einer ungünstigen Prognose vergesellschaftet, da die Patienten meist nur eingeschränkt auf eine Immuntherapie ansprechen. Eine standardisierte Therapie wurde bislang nicht definiert. Kortikosteroide, intravenöse Immunglobuline und Cyclophosphamid kombiniert mit Chemotherapie können helfen, die neurologische Symptomatik zu stabilisieren oder zu verbessern [6].

Symptome einer Enzephalitis können auch in Assoziation mit Oberflächenrezeptorantikörpern wie etwa gegen „contactin-associated protein-like 2“ (CASPR2), den a-Amino-3-hydroxy-5hydroxy-5-methyl-4-isoxazolpropionsäure-Rezeptor (AMPAR), „dipeptidyl-peptidase-like protein-6" (DPPX) oder den $\gamma$-Aminobuttersäure-Rezeptor $A\left(G A B A_{A} R\right)$ auftreten. Patienten mit $\mathrm{GABA}_{\mathrm{A}} \mathrm{R}$-Antikörper entwickeln häufig schwere, oftmals therapieresistente epileptische Anfälle. Im Gegensatz $\mathrm{zu}$ anderen Autoimmunenzephalitiden finden sich häufig multifokale, unilaterale oder bilaterale kortikosubkortikale T2/FLAIR-MRT-Veränderungen, die oft nur eingeschränkt mit der klinischen Symptomatik korrelieren und reversibel sind [2].

\section{Paraneoplastische zerebelläre Degeneration}

Die paraneoplastische zerebelläre Degeneration (PCD) ist klinisch durch ein subakutes Auftreten von Ataxie, Gangstörung, Nystagmus, Dysarthrie, Übelkeit und Erbrechen charakterisiert. Zu Erkrankungsbeginn kann der Liquor entzündliche Veränderungen mit Erhöhung von Zellzahl und Eiweiß sowie intrathekaler Immunglobulinsynthese aufweisen. Die Bildgebung ist $\mathrm{zu}$ Beginn meist unauffällig, zeigt jedoch nach Monaten bis Jahren oftmals eine zunehmende diffuse zerebelläre Atrophie [6]. Bei bis zu $60 \%$ der Patienten mit PCD können klassische onkoneuronale Antikörper nachgewiesen werden, wobei die neurologische Präsentation je nach assoziiertem Antikörper variieren kann. Während Patienten mit Yo-Antikörpern typischerweise an einer relativ isolierten zerebellären Degeneration leiden [6], weisen Patienten mit Anti-HuAntikörpern häufig zusätzlich eine Enzephalomyelitis und Patienten mit RiAntikörpern einen Opsoklonus oder andere Hirnstammsymptome wie Hirnnervenausfälle oder Laryngospasmen auf [6].

\section{》) Die neurologische \\ Präsentation bei PCD kann je nach assoziiertem Antikörper variieren}

Selten können im Rahmen einer PCD auch Oberflächenrezeptorantikörper gefunden werden, wie etwa gegen den „delta/notch-like epidermal growth factor-related receptor“ (Tr/DNER), „P/Qtype voltage-gated calcium channel“ (VGCC) oder $\gamma$-Aminobuttersäure-Rezeptor $B\left(G_{B A} A_{B}\right)$. Bei Patienten mit VGCC-Antikörper und PCD besteht oftmals eine Assoziation mit SCLC; sie können ein Lambert-Eaton-myasthenisches Syndrom (LEMS) entwickeln. VGCC-Antikörper können jedoch auch bei Patienten mit PCD oder LEMS ohne Tumorerkrankung vorkommen. In unklaren Fällen kann die Bestimmung von SOX1-Antikörpern hilfreich sein, um eine paraneoplastische Ursache nachzuweisen [6].

Generell spricht die PCD assoziiert mit onkoneuronalen Antikörpern nur eingeschränkt auf eine Therapie, einschließlich Tumor- und Immuntherapien, an. Die meisten Patienten zeigen eine progrediente Verschlechterung und werden rollstuhlpflichtig. Anders verhält es sich bei PCD-Patienten mit $\mathrm{Tr} /$ DNER-Antikörpern und anderen Ober- 
Hier steht eine Anzeige.

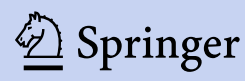


Internist 2018 · 59:151-158 https://doi.org/10.1007/s00108-017-0373-2

(c) Der/die Autor(en) 2018. Dieser Artikel ist eine Open-Access-Publikation.

\section{De Simoni $\cdot$ R. Höftberger}

\section{Paraneoplastische neurologische Syndrome. Eine aktuelle Zusammenfassung}

\section{Zusammenfassung}

Hintergrund. Paraneoplastische neurologische Syndrome (PNNS) sind tumorassoziierte Erkrankungen des Nervensystems, die abseits vom Tumorgeschehen durch eine gestörte Immunreaktion ausgelöst werden. Das Spektrum der PNNS hat sich in den letzten zehn Jahren durch die Entdeckung der Autoimmunenzephalitis assoziiert mit Antikörpern gegen Oberflächen- bzw. synaptische Proteine erweitert, im Gegensatz zu den klassischen PNNS sprechen die betroffenen Patienten meist gut auf eine Immuntherapie an.

Ziel der Arbeit. Im vorliegenden Beitrag werden die wichtigsten PNNS zusammengefasst. Unter Berücksichtigung der neuesten Erkenntnisse auf dem Gebiet der Oberflächenautoimmunität wird auf die speziellen Merkmale der jeweiligen Antikörper eingegangen.

Ergebnisse. Klassische PNNS sind mit Antikörper gegen intrazelluläre Antigene assoziiert (onkoneuronale Antikörper). Sie treten in der Regel vor der Diagnose des Tumorleidens auf und führen zu dessen Entdeckung. Betroffene Patienten sind oft älter und weisen eine ungünstige Prognose auf. Patienten mit Antikörpern gegen Oberflächenproteine können sich ähnlich wie Patienten mit klassischem PNNS präsentieren, nicht immer liegt aber ein Tumor zugrunde. Die Prognose ist deutlich besser, da die Patienten meist gut auf Immuntherapie ansprechen. Einige der Oberflächenantikörper manifestieren sich in Form charakteristischer Syndrome, die dann nach dem entsprechenden Antikörper benannt werden; ein Beispiel ist die Anti-
N-Methyl-D-aspartat-Rezeptor(NMDAR)Enzephalitis. Andere können beträchtliche klinische Gemeinsamkeiten aufweisen und sind schwer voneinander abgegrenzbar, wie etwa bei der limbischen Enzephalitis assoziiert mit $\gamma$-Aminobuttersäure-Rezeptor- $B\left(G A B A_{B} R\right)$ und a-Amino-3-hydroxy-5-hydroxy-5-methyl4-isoxazolpropionsäure-Rezeptor(AMPAR)Antikörpern. Die Diagnose der PNNS ist wichtig, da eine rasche und gezielte Therapie mit einem besseren Outcome der Patienten assoziiert ist.

\section{Schlüsselwörter}

Onkoneuronale Antikörper · NMDAREnzephalitis · Limbische Enzephalitis . Paraneoplastische zerebelläre Degeneration . $\mathrm{GABA}_{\mathrm{B}}$-Rezeptoren

\section{Paraneoplastic neurological syndromes. A current summary}

\section{Abstract}

Background. Paraneoplastic neurological syndromes (PNNS) are remote effects of a tumor and mediated by an altered immune reaction. In the last ten years, the spectrum of PNNS has changed profoundly with the discovery of a new category of neurological diseases that are associated with antibodies against surface or synaptic antigens. In contrast to classical PNNS, patients with surface receptor autoimmunity are often highly responsive to immunotherapy. Objectives. This article provides an update on the most relevant PNNS, focusing on specific syndromes associated with antibodies against classical onconeuronal antigens as well as surface and synaptic proteins.
Results. Classical PNNS are associated with antibodies against intracellular antigens (onconeuronal antibodies). They usually precede the tumor diagnosis and lead to the detection of the neoplasm. Affected patients are often older and have an unfavorable prognosis. Patients with surface receptor autoimmunity can have a similar presentation as classical PNNS; however, the disease is not necessarily triggered by a tumor and patients usually show a good response to treatment. Some surface receptor antibodies might manifest in highly characteristic syndromes and the resulting disease is named after the antibody, such as in anti-N-methyl-Daspartate receptor (NMDAR) encephalitis.
Other antibodies have considerable overlap in their clinical presentation and may be difficult to distinguish, such as in limbic encephalitis associated with GABA(B)R and a-amino-3-hydroxy-5-hydroxy-5-methyl4-isoxazolpropionsäure receptor (AMPAR) antibodies. The diagnosis of the PNNS is important for an early recognition of a tumor and prompt initiation of treatment, which is associated with a better outcome of patients.

\section{Keywords}

Antibodies, onconeuronal · Anti-N-methylD-aspartate receptor encephalitis · Limbic encephalitis · Paraneoplastic cerebellar degeneration · Receptors, GABA-B flächenrezeptorantikörpern. Sie erleiden weniger ausgeprägte Behinderungen und sprechen auf eine Tumor- und/oder Immuntherapie besser an $[2,6]$.

\section{Limbische Enzephalitis}

Die limbische Enzephalitis (LE) ist klinisch durch einen subakuten Kurzzeitgedächtnisverlust, Krampfanfälle, Irritabilität, Depression und kognitiven Abbau gekennzeichnet. In der Bildgebung zeigen sich charakteristischerweise uni- oder bilaterale mesiotemporale FLAIRoder T2-Hyperintensitäten [2]. Die LE kann sowohl im Rahmen klassischer PNNS als auch mit Oberflächenrezeptorantikörpern auftreten.

$\mathrm{Zu}$ den mit LE assoziierten onkoneuronalen Antikörpern gehören Anti-Hu, -Ma2 und -CV2/CRMP5. Die Mehrzahl der Patienten mit Hu-Antikörpern entwickelt im Laufe der Erkrankung weitere Symptome einer diffusen multifokalen Enzephalomyelitis. Patienten mit Anti-CV2/CRMP5-Antikörpern können zusätzlich eine sensomotorische Neuropathie, zerebelläre Ataxie oder Optikusneuropathie entwickeln. Anti-Ma2Antikörper werden meist bei jungen Männern mit Keimzelltumoren des Hodens gefunden und führen $\mathrm{zu}$ LE mit hypothalamischer sowie Hirnstammdysfunktion. Sie können entsprechend Symptome wie Hyperphagie, Hypersomnie, Narkolepsie, Kataplexie oder Endokrinopathien verursachen. Bei älteren Männern und Frauen mit Ma2Antikörpern sind die häufigsten as- 


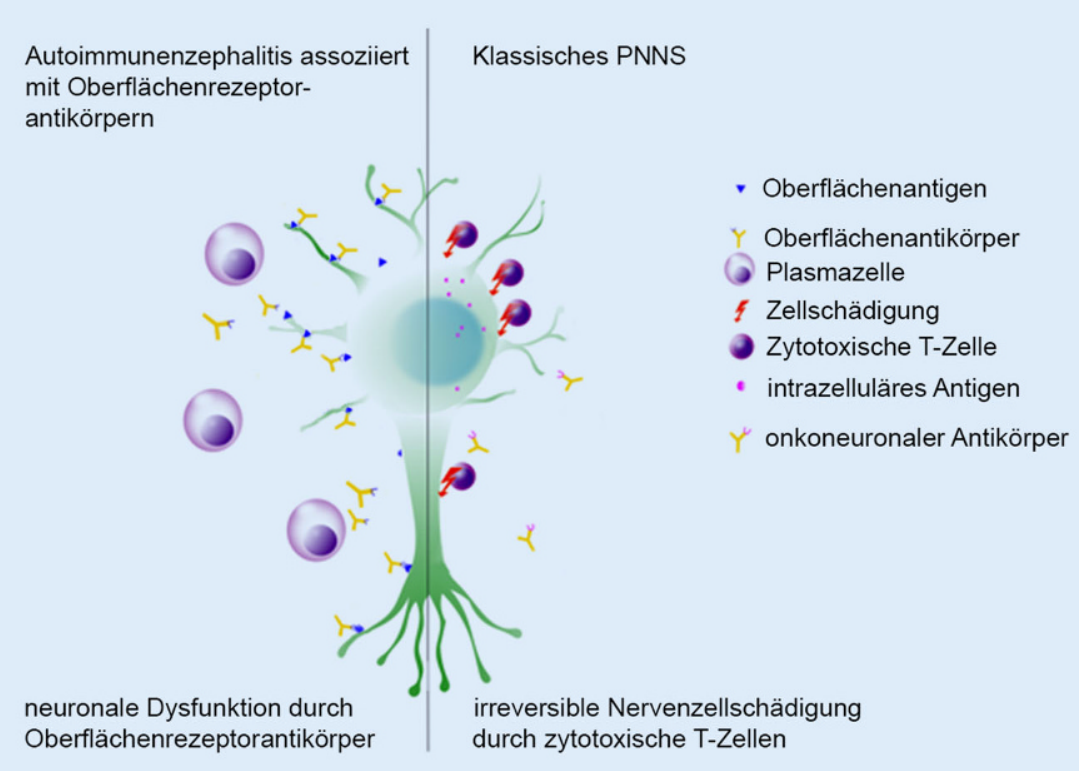

Abb. 1 ॥ Immunmechanismen bei Autoimmunenzephalitis assoziiert mit Oberflächenrezeptorantikörpern und bei klassischem PNNS. PNNS Paraneoplastisches neurologisches Syndrom

soziierten Tumoren nichtkleinzellige Lungenkarzinome („non-small-cell lung cancer" [NSCLC]). LE assoziiert mit onkoneuronalen Antikörpern sprechen in den meisten Fällen nur eingeschränkt auf eine Immun- bzw. Tumortherapie an. Die Ausnahme ist die LE assoziiert mit Ma2-Antikörpern, die sich bei etwa $30 \%$ der Patienten nach Tumor- und Immuntherapie bessert [6].

Die LE kann auch mit Oberflächenrezeptorantikörpern wie Anti-LGI1, -CASPR2, -GABA ${ }_{B}$ R, -AMPAR und -mGluR5 assoziiert sein. Bei all diesen Antikörpern kann eine isolierte LE vorliegen, bei einigen finden sich jedoch noch zusätzliche Symptome, die auf die zugrunde liegende Autoimmunität hinweisen. Beispielsweise findet sich bei LE assoziiert mit LGI1-Antikörpern in $65 \%$ der Fälle eine Hyponatriämie und in $45 \%$ entwickeln sich entweder zu Beginn oder im Laufe der Erkrankung charakteristische faziobrachiale dystone Anfälle [2]. Es konnte gezeigt werden, dass ein signifikanter Teil der idiopathischen, nicht jedoch der paraneoplastischen Fälle von LGI1-Autoimmunenzephalitis mit HLADR7 und HLA-DRB4 assoziiert ist, das Fehlen dieses Haplotyps könnte daher den Verdacht auf eine Tumorerkrankung aufwerfen [2]. Bei der LE assoziiert mit Anti-CASPR2-Antikörpern können
$34 \%$ zerebelläre Symptome zeigen, etwa ein Drittel der Patienten entwickelt ein Morvan-Syndrom, ein komplexes Krankheitsbild, das durch eine periphere Nervenhyperexzitabilität (Faszikulationen, Myokymien, Myoklonie, Tremor), autonome Störungen (Schwitzen, Tachykardie, Hyperthermie, Bluthochdruck), Schlafstörungen, brennende Schmerzen und Enzephalitis gekennzeichnet ist. Bei etwa $20 \%$ finden sich Tumoren (meist Thymome; [2]). Bei Diagnose einer LE sollte in Zusammenschau mit dem zugrunde liegenden Oberflächenrezeptorantikörper eine gezielte Tumorsuche gestartet und rasch eine Immuntherapie eingeleitet werden [7].

\section{Opsoklonus-Myoklonus-Syndrom}

Das Opsoklonus-Myoklonus Syndrom (OMS) ist klinisch durch eine Kombination aus Opsoklonus und arrhythmischem Aktionsmyoklonus, der vor allem den Stamm und die Extremitäten betrifft, charakterisiert [6]. Häufig wird die Erkrankung von Ataxie und Dysarthrie begleitet. In einigen Fällen kann es zu Symptomen einer diffusen Enzephalopathie mit Stupor, Koma und Tod kommen.

Das OMS kann sowohl paraneoplastisch als auch idiopathisch auftreten. Bei
Erwachsenen ist das Syndrom in etwa $39 \%$ der Fälle mit Tumoren wie Lungen(SCLC und NSCLC) oder Mammakarzinomen und Ovarialteratomen assoziiert. In etwa $70 \%$ der Fälle geht das OMS der Tumordiagnose voraus. Patienten mit OMS sind meist älter, mit Ausnahme jener mit Teratomen.

Onkoneuronale Antikörper können etwa bei $11 \%$ der erwachsenen Patienten mit OMS gefunden werden (gegen $\mathrm{Ri}$, Ma2, Zic-4, CV2/CRMP5), Oberflächenrezeptorantikörper bei etwa $17 \%$ (gegen Glycinrezeptor, GABA ${ }_{B} R$, N-Methyl-Daspartat-Rezeptor [NMDAR], DPPX, IgM-Antikörper gegen HNK1 und andere), wobei Glycinrezeptor(GlyR)-Antikörper besonders häufig bei Patienten mit Lungenkarzinomen gefunden wurden [1].

\section{》) Bei jungen Erwachsenen tritt ein OMS meist idiopathisch oder postinfektiös auf}

Eine paraneoplastische Genese des OMS sollte insbesondere bei älteren Erwachsenen, die zusätzlich eine Enzephalopathie oder andere atypische Präsentationen entwickeln, erwogen werden. Erwachsene mit paraneoplastischem OMS haben häufiger Rezidive und ein schlechteres Outcome als idiopathische Fälle. Es wird daher eine möglichst frühe Tumortherapie und aggressive Immuntherapie, beispielsweise mit Rituximab oder Cyclophosphamid, empfohlen. Bei jungen Erwachsenen tritt ein OMS meist idiopathisch oder postinfektiös auf und ist mit einer besseren Prognose vergesellschaftet. In dieser Altersgruppe ist es wichtig, ein Teratom auszuschließen, wobei $\mathrm{Pa}$ tienten mit Teratomen häufig zusätzlich Symptome wie Dysautonomie, Verhaltensauffälligkeiten oder Schwindel aufweisen. Meist können hier jedoch keine auslösenden Antikörper identifiziert werden [1].

\section{Stiff-person-Syndrom-Spektrum}

Das Stiff-person-Syndrom (SPS) ist klinisch durch fluktuierende Muskelsteifigkeit und schmerzhafte Spasmen charakterisiert, die spontan oder durch 
Tab. 2 Hauptmerkmale und assoziierte Tumoren der antikörperassoziierten paraneoplastischen neurologischen Syndrome

\begin{tabular}{|c|c|c|}
\hline Antigene & Assoziierte Tumoren & Hauptmerkmale \\
\hline \multicolumn{3}{|c|}{ Intrazelluläre Antigene } \\
\hline $\mathrm{Hu}$ & $\mathrm{SCLC}$ & $\begin{array}{l}\text { Enzephalomyelitis, PCD, LE, } \\
\text { Hirnstammenzephalitis }\end{array}$ \\
\hline $\mathrm{Ri}$ & Mamma, SCLC & Hirnstammenzephalitis, OMS \\
\hline Yo & Ovar, Mamma & PCD \\
\hline CV2/CRMP5 & SCLC, Thymome & Enzephalomyelitis, Chorea, PCD, LE \\
\hline Amphiphysin & SCLC, Mamma & SPS, Rigidität, Enzephalomyelitis \\
\hline $\mathrm{Ma} 1 / 2$ & Hoden, Adenokarzinome der Lunge & LE, Hirnstammenzephalitis \\
\hline $\mathrm{DNER} / \mathrm{Tr}$ & Morbus Hodgkin & PCD \\
\hline GAD65 & Neuroendokrin (selten) & SPS, zerebelläre Ataxie, LE, Epilepsie \\
\hline \multicolumn{3}{|c|}{ Oberflächenantigene } \\
\hline NMDAR & $\begin{array}{l}\text { Ovarialteratome (gesamt } 40 \% \text {; } \\
18-45 \text { Jahre } 58 \% \text { ) }\end{array}$ & NMDAR-Enzephalitis \\
\hline LGI1 & $<5 \%$ (Thymome) & $\begin{array}{l}\text { LE, faziobrachiale dystone Anfälle, } \\
\text { Hyponatriämie }\end{array}$ \\
\hline CASPR2 & $\begin{array}{l}\text { Thymome (gesamt } 20 \% \text {; bei Morvan- } \\
\text { Syndrom } 20-50 \% \text { ) }\end{array}$ & $\begin{array}{l}\text { LE, Ataxie, Neuromyotonie, Morvan- } \\
\text { Syndrom }\end{array}$ \\
\hline AMPAR & SCLC, Mamma, Thymome (64 \%) & LE \\
\hline $\mathrm{GABA}_{B} \mathrm{R}$ & $\operatorname{SCLC}(50 \%)$ & LE, Ataxie \\
\hline $\mathrm{GABA}_{A} \mathrm{R}$ & $\begin{array}{l}\text { Hämatologische Tumoren, Thymome } \\
(25 \%)\end{array}$ & $\begin{array}{l}\text { Enzephalitis, Status epilepticus, Anfäl- } \\
\text { le }\end{array}$ \\
\hline mGluR1 & Hämatologische Tumoren (30-40 \%) & Zerebelläre Ataxie \\
\hline mGluR5 & Morbus Hodgkin & LE \\
\hline DPPX (Kv4.1) & Hämatologische Tumoren (selten) & $\begin{array}{l}\text { Gedächtnisstörungen, Enzephalitis, } \\
\text { Myoklonien, Tremor, Anfälle, PERM }\end{array}$ \\
\hline Glycinrezeptor & Lungentumoren (selten) & SPS, PERM, OMS \\
\hline P/Q-Typ-VGCC & $\mathrm{SCLC}$ & $\begin{array}{l}\text { Ataxie, Lambert-Eaton-myastheni- } \\
\text { sches Syndrom }\end{array}$ \\
\hline
\end{tabular}

AMPAR a-Amino-3-hydroxy-5-hydroxy-5-methyl-4-isoxazolpropionsäure-Rezeptor; CASPR2 "contactin-associated protein-like 2"; $D P P X_{\text {" }}$ dipeptidyl-peptidase-like protein-6"; $G A B A_{A} R$ Y-Aminobuttersäure-Rezeptor A; $G A B A_{B} R$ Y-Aminobuttersäure-Rezeptor B; GAD65 "glutamic acid decarboxylase 65"; LE limbische Enzephalitis; $m$ GluR1 metabotroper Glutamatrezeptor 1; $m$ GluR5 metabotroper Glutamatrezeptor 5; NMDAR N-Methyl-D-aspartat-Rezeptor; OMS Opsoklonus-Myoklonus-Syndrom; $P C D$ paraneoplastische zerebelläre Degeneration; $P E R M$ progressive Enzephalomyelitis mit Rigidität und Myoklonus; SCLC "small cell lung cancer" (kleinzelliges Lungenkarzinom); SPS Stiffperson-Syndrom; Tr/DNER „delta/notch-like epidermal growth factor-related receptor"; VGCC „Voltage-gated calcium channel" (spannungsabhängiger Kalziumkanal)

verschiedene Stimuli ausgelöst werden. Partielle oder segmentale Formen der Erkrankung wie das Stiff-limb-Syndrom oder auch schwerere Verlaufsformen wie die progressive Enzephalomyelitis assoziiert mit Rigidität und Myoklonien (PERM) werden üblicherweise als Teil des SPS-Spektrums betrachtet. Bei etwa zwei Drittel können zugrunde liegende Autoantikörper gefunden werden, am häufigsten gegen "glutamic acid decarboxylase 65" (GAD65; $43 \%$ ) und GlyR (etwa $20 \%$ ), selten gegen andere Antigene wie Amphiphysin, Gephyrin, DPPX oder $\mathrm{GABA}_{\mathrm{A}} \mathrm{R}$ [5]. Nur bei etwa 2-3\% der Patienten handelt es sich dabei um paraneoplastische Formen, dann meist in Assoziation mit Anti-AmphiphysinAntikörpern bei Patienten mit Mammakarzinomen, Lungenkarzinomen oder seltener Hodgkin-Lymphomen. AntiGAD65-Antikörper können selten in Assoziation mit Tumoren auftreten, meist der Lunge oder des Thymus. Das Risiko einer paraneoplastischen Genese steigt jedoch bei älteren Patienten, männlichem Geschlecht und wenn zusätzlich Oberflächenrezeptorantikörper wie etwa gegen $\mathrm{GABA}_{\mathrm{B}} \mathrm{R}$ auftreten [2]. Patienten mit Anti-GAD65-Antikörpern weisen eine schlechtere Prognose auf als solche mit GlyR-Antikörpern.

\section{Anti-NMDAR-Enzephalitis}

Die Anti-NMDAR-Enzephalitis ist nach derzeitigem Wissensstand die häufigste unter den antineuronalen Autoimmunenzephalitiden. In einer Studie mit 577 Patienten wurde bei $38 \%$ (97\% davon weibliche Patienten) eine Tumorerkrankung beobachtet, in den allermeisten Fällen Ovarialteratome (94\%), selten andere Tumoren (extraovarielle Teratome $2 \%$, andere Tumoren $4 \%$ ). Am häufigsten waren Frauen bzw. Mädchen im Alter zwischen 12 und 45 Jahren betroffen [2].

Die Erkrankung zeigt charakteristischerweise einen stadienhaften Verlauf. $\mathrm{Zu}$ Beginn entwickeln etwa die Hälfte der Patienten eine Prodromalsymptomatik mit Fieber, Krankheitsgefühl, Kopfschmerzen oder Durchfall. Etwa 1-2 Wochen später treten komplexe neuropsychiatrische Symptome wie Agitiertheit, Psychosen (wahnhafte Vorstellungen, manisch-depressive Episoden, Aggressivität), Katatonie, Gedächtnisstörungen, Sprachreduktion, abnorme Bewegungen oder epileptische Anfälle auf. Bei etwa der Hälfte der Patienten kommt es schließlich $\mathrm{zu}$ autonomen Funktionsstörungen (Hypoventilation, Hyperthermie, Tachykardie) und Koma, sodass eine intensivmedizinische Behandlung notwendig wird. Die Mortalität wird auf $7 \%$ geschätzt. Der Liquor zeigt häufig eine erhöhte Zellzahl, erhöhtes Protein und eine intrathekale Immunglobulinsynthese. Das EEG ist bei über $90 \%$ der Patienten pathologisch, in etwa einem Drittel der Fälle kann ein sogenanntes „extrem delta brush pattern" nachgewiesen werden, das zwar nicht als absolut spezifisch für die NMDAR-Enzephalitis gilt, jedoch dringend an die Differenzialdiagnose denken lassen sollte. MRT-Auffälligkeiten sind nur bei etwa $33 \%$ der Patienten nachweisbar und präsentieren sich meist in Form unspezifischer kortikaler oder subkortikaler T2/FLAIR-Abnormitäten.

Die Diagnose der NMDAR-Enzephalitis kann durch Detektion von IgGAntikörpern gegen ein spezifisches extrazelluläres Epitop der GluN1-Unter- 
einheit des Rezeptors bestätigt werden. Diese Antikörper sind spezifisch für die NMDAR-Enzephalitis und sollten nicht mit Antikörpern gegen andere Untereinheiten des Rezeptors oder mit anderen Immunglobulinklassen verwechselt werden. Patienten mit NMDAR-Enzephalitis sprechen meist gut auf eine Immuntherapie und auf die Entfernung eines eventuell vorhandenen Tumors an. Dabei ließ sich zeigen, dass durch eine möglichst frühe sowie aggressive Immuntherapie, beispielsweise mit Rituximab oder Cyclophosphamid, das Outcome verbessert bzw. die Rezidivrate reduziert werden kann [2].

\section{Krankheitsbilder des peripheren Nervensystems}

\section{Paraneoplastische sensorische Neuronopathie}

Der Begriff der paraneoplastischen sensorischen Neuronopathie (PSN) wurde eingeführt, um eine primäre Schädigung der Nervenzellen der sensiblen Hinterstrangganglien $\mathrm{zu}$ beschreiben. Das Krankheitsbild ist charakterisiert durch einen akuten bis subakuten Beginn mit hochgradigem Verlust der Hinterstrangqualitäten mit Parästhesien, Schmerzen und sensorischer Ataxie. Die sensiblen Ausfälle sind meist asymmetrisch und multifokal, die oberen Extremitäten sind häufig betroffen. Auch die Hirnnerven können betroffen sein, mit daraus resultierender Hypästhesie des Gesichts, sensorineuraler Hypakusis oder Geschmacksverlust. In der elektroneurographischen Untersuchung zeigt sich ein Ausfall der sensiblen Nervenleitgeschwindigkeit (NLG), während die motorischen NLG erhalten sind. In der überwiegenden Mehrheit der Fälle tritt die Neuronopathie vor der Tumordiagnose auf. Der häufigste zugrunde liegende Tumor ist das SCLC. Die PSN kann aber auch bei Adenokarzinomen, Lymphomen oder Thymomen auftreten [6]. Onkoneuronale Antikörper finden sich in etwa $80 \%$ der PSN-Fälle, zumeist Anti-Hu- oder Anti-CV2/CRMP5-Antikörper.

\section{Fazit für die Praxis}

- Die Diagnose von PNNS setzt eine gute neurologische Abklärung sowie die Kenntnis der spezifischen Krankheitsbilder voraus.

- Das PNNS tritt häufig vor Nachweis eines Tumors auf.

- Bei onkologischen Patienten müssen differenzialdiagnostisch unter anderem Metastasen sowie toxische, metabolische, infektiöse oder vaskuläre Ursachen ausgeschlossen werden.

- Abhängig von den Immunmechanismen (intrazelluläre vs. Oberflächenrezeptorantikörper) sind unterschiedliche Therapieerfolge zu erwarten.

- Die klassischen PNNS sprechen meist nur eingeschränkt auf eine Immuntherapie an. Der rasche Einsatz einer immunsupprimierenden Therapie kann allerdings helfen, die neurologische Symptomatik zu stabilisieren oder zu verbessern.

- Bei der Autoimmunenzephalitis assoziiert mit Oberflächenantikörpern ist die Immuntherapie dagegen mit hohen Erfolgsaussichten verbunden.

- Im praktischen Alltag sollte der Einsatz einer aggressiveren Immuntherapie bei Patienten mit PNNS und gleichzeitiger Tumortherapie mit den behandelnden Onkologen sorgfältig geplant und abgestimmt werden.

\section{Korrespondenzadresse}

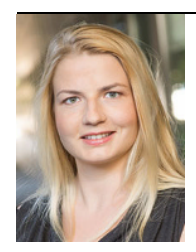

Dr. D. De Simoni

Klinisches Institut für Neurologie, Medizinische Universität Wien Währinger Gürtel 18-20, 1090 Wien, Österreich desiree.desimoni@ meduniwien.ac.at

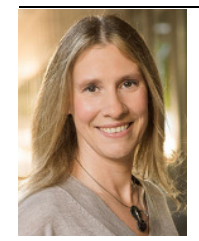

Assoc. Prof. PD Dr. R. Höftberger Klinisches Institut für Neurologie, Medizinische Universität Wien Währinger Gürtel 18-20, 1090 Wien, Österreich romana.hoeftberger@ meduniwien.ac.at
Hier steht eine Anzeige. Springer 
Funding. Open access funding provided by Medical University of Vienna.

\section{Einhaltung ethischer Richtlinien}

Interessenkonflikt. D. De Simoni und R. Höftberger geben an, dass kein Interessenkonflikt besteht.

Dieser Beitrag beinhaltet keine von den Autoren durchgeführten Studien an Menschen oder Tieren.

Open Access Dieser Artikel wird unter der Creative Commons Namensnennung 4.0 International Lizenz (http://creativecommons.org/licenses/by/4.0/deed. de) veröffentlicht, welche die Nutzung, Vervielfältigung, Bearbeitung, Verbreitung und Wiedergabe in jeglichem Medium und Format erlaubt, sofern Sie den/die ursprünglichen Autor(en) und die Quelle ordnungsgemäßnennen, einen Linkzur Creative Commons Lizenz beifügen und angeben, ob Änderungen vorgenommen wurden.

\section{Literatur}

1. Armangue T, Sabater L, Torres-Vega E et al (2016) Clinical and immunological features of opsoclonus-myoclonus syndrome in the era of neuronal cell surface antibodies. JAMA Neurol 73:417-424

2. Dalmau J, Geis C, Graus F (2017) Autoantibodies to synaptic receptors and neuronal cell surface proteins in autoimmune diseases of the central nervous system. Physiol Rev 97:839-887

3. Graus F, Delattre JY, Antoine JC et al (2004) Recommended diagnostic criteria for paraneoplastic neurological syndromes. J Neurol Neurosurg Psychiatr 75:1135-1140

4. Graus F, Titulaer MJ, Balu R et al (2016) A clinical approach to diagnosis of autoimmune encephalitis. Lancet Neurol 15:391-404

5. Martinez-Hernandez E, Arino H, Mckeon A et al (2016) Clinical and immunologic investigations in patients with stiff-person spectrum disorder.JAMA Neurol 73:714-720

6. Rosenfeld MR, Dalmau JO (2012) Paraneoplastic disorders of the CNS and autoimmune synaptic encephalitis. Continuum (Minneap Minn) 18:366-383

7. Titulaer MJ, Soffietti R, Dalmau J et al (2011) Screening for tumours in paraneoplastic syndromes: report of an EFNS task force. Eur J Neurol 18:19-e13

\section{Ausschreibung \\ Oskar-Medizin-Preis 2018}

Die Stiftung Oskar-Helene-Heim fördert die Wissenschaft und Forschung auf dem Gebiet der Medizin. Als besonderes Förderprojekt verleiht die Stiftung jährlich den mit $50.000 €$ dotierten Oskar Medizin-Preis. Mit diesem Medizinpreis werden hervorragende Leistungen gewürdigt und die Weiterführung von Forschungen unterstützt.

Im Jahr 2018 ist der Oskar Medizin-Preis auf dem Gebiet der

Gastroenterologie, Verdauungsund Stoffwechselkrankheiten mit dem Thema „Adipositas"

ausgeschrieben.

Adipositas stellt ein globales Problem dar: Weltweit ist derzeit jeder dritte Mensch übergewichtig oder fettleibig, auch in Deutschland. Damit ist das krankhafte Übergewicht international zu einer gesamtgesellschaftlich relevanten, gesundheitlichen Bedrohung geworden. Besonders der rasche Anstieg der Adipositas bei Kindern und Jugendlichen bereitet große Sorgen. Die mit dem Übergewicht steigenden Risiken für Krankheiten wie Herzinfarkt, Schlaganfall und Bluthochdruck, aber auch die psychischen und sozialen Folgen der Fettleibigkeit erfordern es, dem Problem größte Aufmerksamkeit zu schenken. Die Spanne der Aspekte reicht dabei von der Soziologie und Epidemiologie über die Ätiologie, Pathophysiologie und Ernährung bis zur psychologischen, bewegungstherapeutischen, diätetischen, medikamentösen und chirurgischen Behandlung der Adipositas. Zur Förderung dieser Forschung soll mit dem Oskar Medizinpreis 2018 ein/e habilitierte/r Mediziner/in oder Wissenschaftler/in ausgezeichnet werden, die/der einen relevanten Beitrag zu einem der genannten Teilaspekte des Themas

\section{"Adipositas"}

in der Grundlagen- und/oder klinischen Forschung leistet.
Fachübergreifende Arbeitsgruppen sind ebenfalls zur Bewerbung zugelassen

\section{Stiftung Oskar Helene Heim

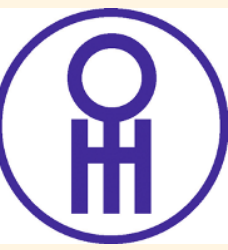

Der prämierte Erkenntnisgewinn soll für eine breite Öffentlichkeit vermittelbar sein.

- Dem Antrag sollen der Lebenslauf, die wichtigsten Arbeiten aus den letzten drei Jahren zum Thema "Prävention durch Bewegung" (Arbeiten, die bereits eine anderweitige Prämierung erhalten haben, sind ausgeschlossen) sowie eine inhaltliche Zusammenfassung der Forschungsergebnisse ergänzt um die weitere Forschungsplanung beigefügt werden.

- Das Preisgeld ist für Forschungszwecke nach der freien Entscheidung des Preisträgers zu verwenden. Der Preisträger hat der Stiftung diese Verwendung in geeigneter Weise zu belegen.

- Der Antrag ist bis zum 31. Mai 2018 bei der Stiftung Oskar-Helene-Heim, Walterhöferstr. 11, 14165 Berlin einzureichen (info@stiftung-ohh.de).

Weitere Informationen erteilt der Geschäftsführer der Stiftung, Thomas Höhn (thomas.hoehn@helios-gesundheit.de, Tell. 030/8102-1104)

Zweck der Stiftung Oskar-Helene-Heim ist die Förderung von Wissenschaft und Forschung in der Medizin, insbesondere der Orthopädie, der Lungenheilkunde, Gastroenterologie und Viszeralchirurgie sowie der Orthopädietechnik. Zu diesem Zweck unterstützt die Stiftung Forschungsprojekte oder sonstige gemeinnützige gesundheitsfördernde Vorhaben, verleiht Stipendien und vergibt jährlich den Oskar-Medizinpreis und die Helene-Medaille.

Quelle: Stiftung Oskar-Helene-Heim, www.stiftung-ohh.de 